\title{
Crocin suppresses multidrug resistance in MRP overexpressing ovarian cancer cell line

\author{
Shadi Mahdizadeh ${ }^{1}$, Gholamreza Karimi ${ }^{2}$, Javad Behravan ${ }^{3,4}$, Sepideh Arabzadeh ${ }^{3}$, Hermann Lage ${ }^{5}$ \\ and Fatemeh Kalalinia ${ }^{3,6^{*}}$
}

\begin{abstract}
Background: Crocin, one of the main constituents of saffron extract, has numerous biological effects such as anti-cancer effects. Multidrug resistance-associated proteins 1 and 2 (MRP1 and MRP2) are important elements in the failure of cancer chemotherapy. In this study we aimed to evaluate the effects of crocin on MRP1 and MRP2 expression and function in human ovarian cancer cell line A2780 and its cisplatin-resistant derivative A2780/RCIS cells.

Methods: The cytotoxicity of crocin was assessed by the MTT assay. The effects of crocin on the MRP1 and MRP2 mRNA expression and function were assessed by real-time RT-PCR and MTT assays, respectively.

Results: Our study indicated that crocin reduced cell proliferation in a dose-dependent manner in which the reduction in proliferation rate was more noticeable in the A2780 cell line compared to A2780/RCIS. Crocin reduced MRP1 and MRP2 gene expression at the mRNA level in A2780/RCIS cells. It increased doxorubicin cytotoxicity on the resistant A2780/RCIS cells in comparison with the drug-sensitive A2780 cells.

Conclusion: Totally, these results indicated that crocin could suppress drug resistance via down regulation of MRP transporters in the human ovarian cancer resistant cell line.
\end{abstract}

Keywords: Crocin, Multidrug resistance, MRP1, MRP2, A2780, A2780/RCIS

\section{Background}

Cancer is a leading cause of death in the world that broadly affects more and less economically developed countries [1]. Using different chemotherapy regimens is a common method in cancer treatment, but there is some limitation on the effectiveness of chemotherapy that leads to poor therapeutic results [2]. One of the most important reasons of treatment failure during chemotherapy is the multidrug resistance (MDR) phenomenon. The MDR tumors are resistant to chemotherapeutic agents which are structurally and functionally different from the initial anticancer drug. Typical MDR occurs through overexpression of the membrane efflux proteins that pump anticancer drugs out of the cells [3]. These pharmaceutical transporter proteins belong to the ATP-binding cassette transporters family

\footnotetext{
*Correspondence: kalaliniaf@mums.ac.ir

${ }^{3}$ Biotechnology Research Center, School of Pharmacy, Mashhad University of Medical Sciences, P. O. Box 91775-1365, Mashhad, Iran

${ }^{6}$ Medical Genetic Research Center, Mashhad University of Medical Sciences,

Mashhad, Iran

Full list of author information is available at the end of the article
}

(ABC family) $[4,5]$. One important class of the $A B C$ family is the human multidrug resistance-associated protein (MRP) family which contains seven members. Several members of the MRP family especially MRP1 and MRP2 are involved in the detoxification and protection of the host against xenobiotic materials. They are also assumed to cause drug resistance through their ability in transporting a wide range of anticancer drugs out of the cells and their presence in many different types of tumors [6].

In the last decades, different research explored new botanical candidates with potential anti-cancer effects that has opened a window to developing safer and more effective anti-cancer therapies $[7,8]$. Crocus sativus is a plant of the Iridaceae family. Stigmas of Crocus sativus flowers (saffron) contain various chemical substances [9]. Crocin is a major glycosylated carotenoid found in saffron [10] that has various pharmacological effects like protecting the myocardial cell against hypoxia damage [11], antioxidant [12, 13], anti-atherosclerosis 
$[14,15]$, antidepressant [16] and anti-inflammatory effects $[17,18]$. In addition, different studies have shown anticancer activities of crocin against human leukemia, breast, colorectal, and bladder cancer cell lines [19-23]. Based on these facts, it is expected that crocin could potentially be used clinically for the prevention and treatment of cancer in the near future.

It has shown that crocin inhibits Lipopolysaccharides (LPS)-induced nitric oxide (NO) release from brain microglial cells and reduces the LPS-stimulated productions of tumor necrosis factor-alpha, interleukin-1 beta, and intracellular reactive oxygen species, which effectively cause decreased NF-kappa B activation [17, 24]. On the other hand, it has been previously showed that sulindac, the nonsteroidal anti-inflammatory drug, generates oxidative stress via induction of reactive oxygen species (ROS) production, which finally leads to the higher expression of MRP1 and MRP3 in human colorectal cancer cell lines [25]. These evidences suggest that crocin might affect the protein expression of MDR proteins. In the present study, we aimed to evaluate the effects of crocin on the expression and function of MRP1 and MRP2 in the human ovarian carcinoma cell lines A2780 and its cisplatin-resistant derivative A2780/ RCIS cells (MRP2-overexpressing cell line).

\section{Methods \\ Materials}

Fetal bovine serum (FBS) and RPMI 1640 with L-glutamine were purchased from Gibco (USA) and Biosera (UK), respectively. MTT, DMSO, trypan blue, doxorubicin and penicillin G/streptomycin were obtained from Sigma-Aldrich (Germany). Crocin was generously provided by Dr. Seyed Ahmad Mohajeri (Pharmaceutical Research Center, Mashhad University of Medical Sciences, Iran). RNA tripure isolation kit was obtained from Roche Applied Science, Germany and Real-time EXPRESS One-Step SYBR GreenER ${ }^{\text {тм }}$ Kit was purchased from Invitrogen, USA. The MRP-overexpressing, cisplatinresistant ovarian cancer cell line, A2780/RCIS and its parental cisplatin sensitive cell line A2780 were generously provided by Professor Herman Lage (Molecular Pathology Department, Charite Campus Mitte, Berlin, Germany).

\section{Preparation of the crocin solution}

Total crocin was extracted and crystallized from saffron stigmas and its purity was tested with HPLC and was more than $96 \%$ [26]. Crocin was dissolved in DMSO (dimethyl sulfoxide) and PBS to a final concentration of $1024 \mathrm{mM}$ and stored at $-20{ }^{\circ} \mathrm{C}$. The drug was freshly diluted to its final concentration $(10,20$,
$40,60,80$ and $100 \mu \mathrm{M})$ in culture medium prior to the start of each experiment.

\section{Cell culture and treatment}

Cells were cultured in RPMI-1640 contained FBS $10 \%(\mathrm{v} / \mathrm{v})$, penicillin $(100 \mathrm{U} / \mathrm{mL})$, and streptomycin $(100 \mu \mathrm{g} / \mathrm{mL})$ at $37{ }^{\circ} \mathrm{C}$ in humidified air containing CO2 $5 \%$. For MTT and real-time PCR studies, ovarian cancer cells were incubated for 4-72 h with crocin $(0-100 \mu \mathrm{M})$. For MRP activity analysis, all cell lines were co-treated with different concentrations of crocin $(0-100 \mu \mathrm{M})$ and doxorubicin $(0-500 \mathrm{nM})$ for 4-72 h. This study was obtained the approval of the Research Ethics Committee of Mashhad University of Medical Sciences (code No: IR.MUMS.REC.1390.301).

\section{MTT cytotoxicity assay}

Drug sensitivity of the A2780 cell line and drug-resistant cell line A2780/RCIS were confirmed by MTT assay. Cells were seeded at an initial density of $10^{4}$ cells/well in 96-well plates. The plates were incubated at $37{ }^{\circ} \mathrm{C}$ in a $5 \%$ CO2-supplemented atmosphere for $24 \mathrm{~h}$. Subconfluent cells were treated with different concentrations of crocin and doxorubicin in a final volume of $100 \mu \mathrm{l}$ of standard growth medium in each well. The control wells had DMSO in the growth medium at equal volumes to those used for the test compounds. Cell viability was measured after 4-72 h, using 3-(4, 5-dimethylthiazol-2-yl)-2, 5-diphenyl tetrazolium bromide (MTT). The reduced MTT dye was solubilized with DMSO (100 $\mu \mathrm{l} /$ well) and absorbance was determined on an ELISA plate reader (BioTek, Bad Friedrichshall, Germany) with a test wavelength of $550 \mathrm{~nm}$ and a reference wavelength of $630 \mathrm{~nm}$. Each experiment was performed in triplicate and was repeated at least three times. The percentage of cell proliferation was calculated using the ratio of $\mathrm{OD}_{\text {test }} / \mathrm{OD}_{\text {control }}$.

\section{Real-Time RT-PCR}

Total cellular RNA was extracted using tripure isolation reagent. The total amount of RNA was measured using a NanoDrop 1000 spectrophotometer (Thermo Fisher Scientific, Wilmington, DE) and the acceptable purity was in the range of 1.8-2.2 for the A260/A230 and A260/A280 ratios. Real-time RT-PCR was performed to measure the expression levels of MRP1 and MRP2 in ovarian cancer cell lines using the EXPRESS One-Step SYBR GreenER ${ }^{\mathrm{TM}}$ Kit and real-time cycler Mx3000P $\mathrm{P}^{\mathrm{TM}}$ Stratagen (Stratagen, USA). The primers had the following sequences: MRP1: 5'-GTGTTTCTGGTCAGCC CAACT-3' (forward) and 5'-TTGGATCTCAGGATGG CAGG-3' (reverse); MRP2, 5' -AGCAGCCATAGAGCT GGCCCTT-3' (forward) and 5'-AGCAAAACCAGGA GCCATGTGCC-3' (reverse); $\beta$-actin: 5'-TCATGAAG 
TGTGACGTGGACATC-3' (forward) and 5' -CAGGA GGAGCAATGATCTTGATCT-3' (reverse). Reactions were performed with an initial cDNA synthesis step at $50{ }^{\circ} \mathrm{C}$ for $5 \mathrm{~min}$, followed by the denaturation step at $95{ }^{\circ} \mathrm{C}$ for $2 \mathrm{~min}$ and PCR amplification cycles (40 cycles at $95{ }^{\circ} \mathrm{C}$ for $15 \mathrm{~s}, 60{ }^{\circ} \mathrm{C}$ for $1 \mathrm{~min}$ ). Relative expression levels for MRP1 or MRP2 were normalized to the $\beta$-actin by the MxPro-Mx3000P system. The relative expressions of MRP genes were reported as the target/reference ratio of the treated samples divided by the target/reference ratio of the untreated control sample.

\section{Statistical analysis}

Results (mean $\pm \mathrm{SD}$ ) were reported in three independent stages. Statistical analyses were performed by SPSS version 16.0 using ANOVA, with the Tukey's post-hoc to show significant differences between the data and $p$ values $<0.05$ were considered significant.

\section{Results}

Effect of crocin on the proliferation rate of A2780 cancer cell lines

To investigate the effects of crocin on the cell survival of ovarian cancer cells, A2780 cells were incubated in the presence or absence of various concentrations of crocin $(0-100 \mu \mathrm{M})$ for $4,24,48$ and $72 \mathrm{~h}$ and then subjected to MTT cytotoxicity assay. Crocin showed inhibitory effects on the cell growth rate of A2780 cells in a concentration and time-dependent manner (Fig. 1a). Crocin exhibited a similar inhibitory pattern with less potency in A2780/RCIS (Fig. 1b), in which treatment with $60-100 \mu \mathrm{M}$ and 80 $100 \mu \mathrm{M}$ of crocin significantly reduced the survival of A2780 and A2780/RCIS, respectively ( $P<0.05$ vs. control).
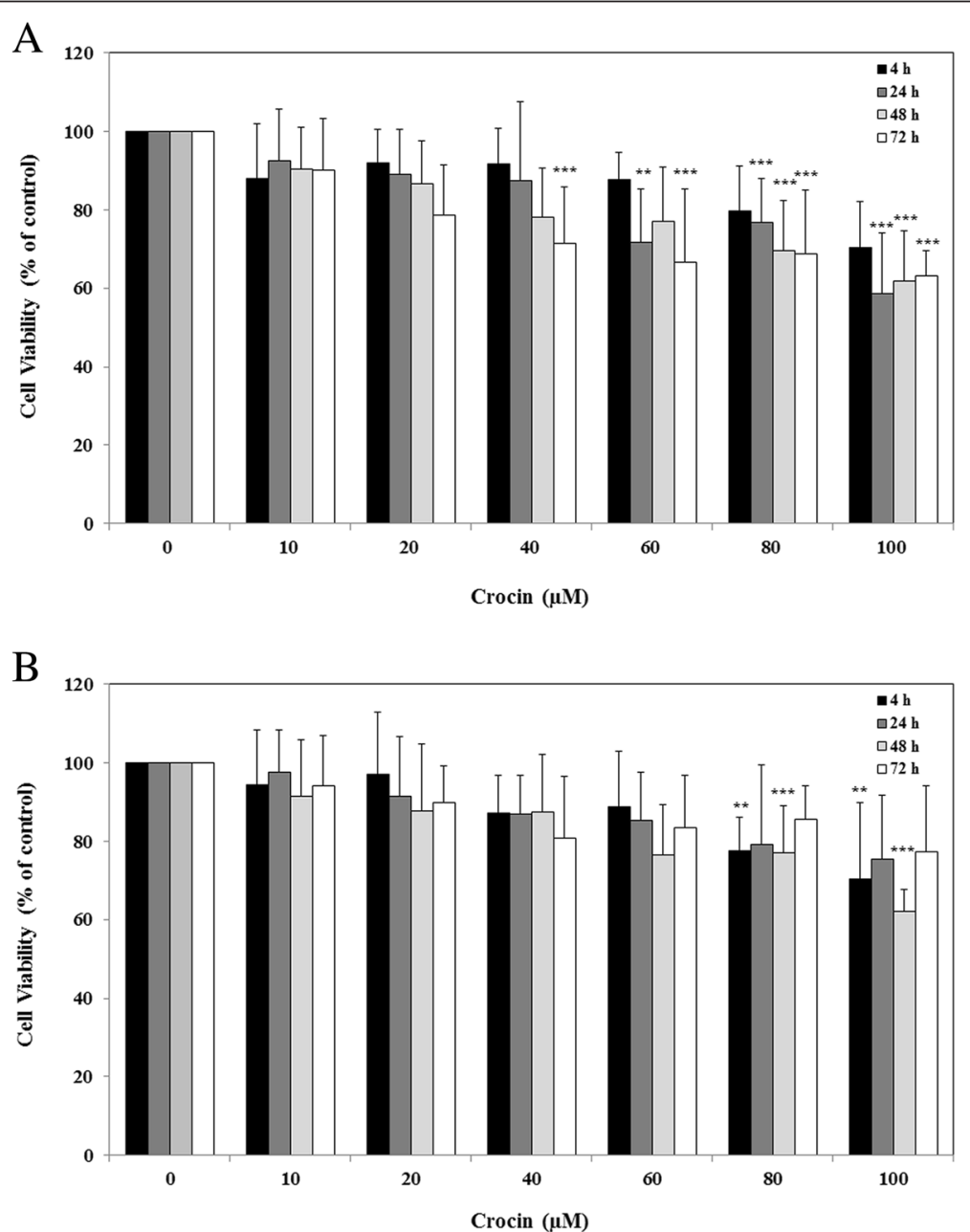

Fig. 1 The effects of crocin on cell viability of A2780 (a) and A2780/RCIS (b) cell lines. The cells were incubated with various concentrations of crocin at $37^{\circ} \mathrm{C}$ for $4,24,48$ and $72 \mathrm{~h}$. Cell viability was measured by the MTT assay. Each experiment was repeated independently three times in triplicate tests and data are shown as mean $\pm \mathrm{SD}$. ${ }^{*} P \leq 0.05 ;{ }^{* *} P \leq 0.01 ;{ }^{* *} P \leq 0.001$ 
Expression of MRP1 and MRP2 in A2780 cell lines

Real-time RT-PCR was used to assess the basic level of the mRNA expression of MRP1 and MRP2 in cisplatinresistant A2780/RCIS cells and sensitive parental A2780 cell line. As shown in Fig. 2a, the MRP1 mRNA level in the A2780/RCIS cell line was 1.29 times more than its expression level in A2780 cells. Also, the results showed that the expression level of MRP2 mRNA in the resistant cell line A2780/RCIS was about 13 times more than the MRP2 mRNA level in parental A2780 cells (Fig. 2b).

\section{Effect of crocin on MRP1 and MRP2 gene expression}

To evaluate the expression of MRP1 and MRP2 mRNA after treatment with crocin, the cells were treated with different concentrations of crocin $(0-100 \mu \mathrm{M})$ for 4 and $48 \mathrm{~h}$. Expression of MRP transporters was studied using Real-time RT-PCR. The results showed that crocin significantly reduced the expression level of MRP1 (up to about $50 \%$ ) in A2780/RCIS cells at 48 h (Fig. 3a). Similarly, crocin could significantly reduce MRP2 mRNA expression (up to $60 \%$ ) in cisplatin-resistant A2780/RCIS cells in a time dependent manner in compared with the control level (Fig. 3b).

\section{Effect of crocin on multi-drug resistance protein activity} In order to evaluate the effects of crocin on MRP transporters activity, sensitivity of tested cell lines to doxorubicin in the presence or absence of crocin were studied. At first it was necessary to find the $\mathrm{IC}_{50}$ of doxorubicin on A2780 and A2780/RCIS cells. For this purpose, cells were treated with different concentrations of doxorubicin (0-500 nM) for 4, 24, 48, and $72 \mathrm{~h}$. Doxorubicin significantly reduced the proliferation rate of A2780 (Fig. 4a) and A2780/RCIS cells (Fig. 4b) in a concentration and time-dependent manner. These antiproliferative effects of doxorubicin were with a higher intensity in the parental cisplatin-sensitive cell line A2780 in comparison with the resistant cell line A2780/ RCIS.

To test the combining effects of crocin and doxorubicin on the cell survival of A2780 cell lines, 30 different combinations $(0-100 \mu \mathrm{M}$ of crocin with $0-500 \mathrm{nM}$ of doxorubicin) were evaluated using MTT assay. The combinatorial effects on cell survival were analyzed after 4, 24, 48, and $72 \mathrm{~h}$ incubation. There were no significant differences in A2780 cell viabilities between all crocin + doxorubicin concentrations and controls with the same amount of doxorubicin (data not shown). On the other hand, different concentrations of crocin could increase the percentage of doxorubicin cytotoxicity in A2780/ RCIS, almost all in a time and concentration-dependent manner (Table 1). While crocin could not change the A2780/RCIS cell sensitivity to doxorubicin in $4 \mathrm{~h}$, there were significant differences between the $\mathrm{IC}_{50}$ of samples that were treated with crocin + doxorubicin and $\mathrm{IC}_{50}$ of controls that were with the same amount of doxorubicin after $48 \mathrm{~h}(144 \mathrm{nM}$ vs. $349 \mathrm{nM}, P<0.001)$ and $72(162 \mathrm{nM}$ vs. $326 \mathrm{~nm}, P<0.01)$.

\section{Discussion}

Multidrug resistance (MDR) is one of the most important reasons for the insufficient effectiveness of chemotherapy drugs in cancer treatment. A major mechanism involved in MDR, is the presence of some of the ATPbinding cassette transporters (ABC) like Multidrug resistance-associated proteins 1 and 2 (MRP1 and MRP2),
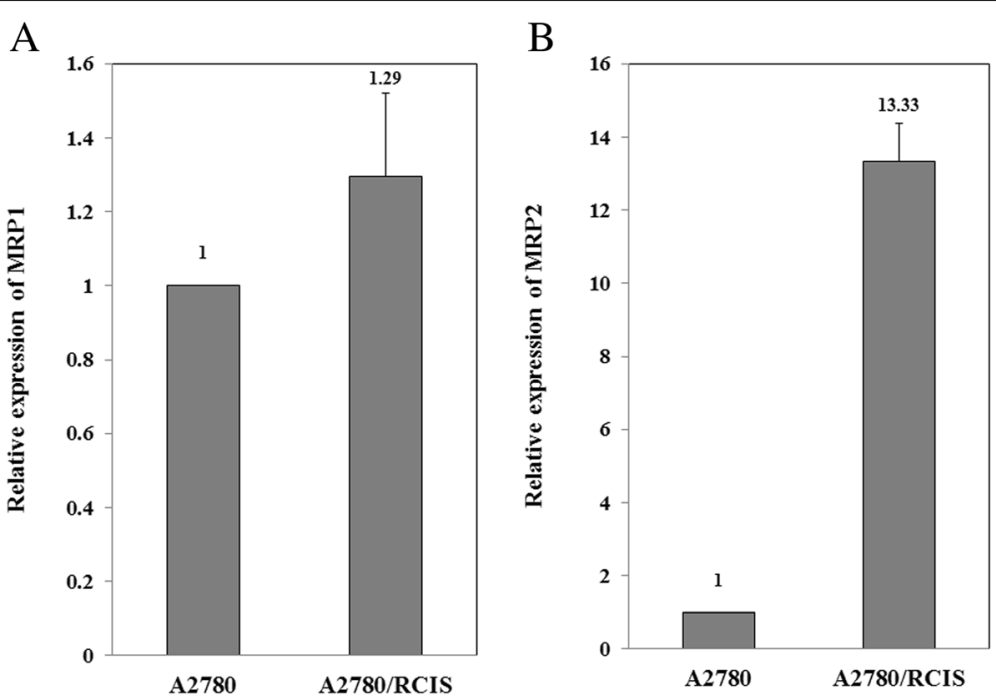

Fig. 2 Basal expressions of MRP1 (a) and MRP2 (b) mRNA in ovarian cancer cell lines were studied by real-time RT-PCR. The MRP mRNA level is compared with the drug-resistant cell line A2780/RCIS and parental drug-sensitive cell line A2780. Real-time RT-PCR analysis was performed on total RNA extracted from cells. Values were normalized to the $\beta$-actin content of samples and expressed as mean \pm SD $(n=3)$. 

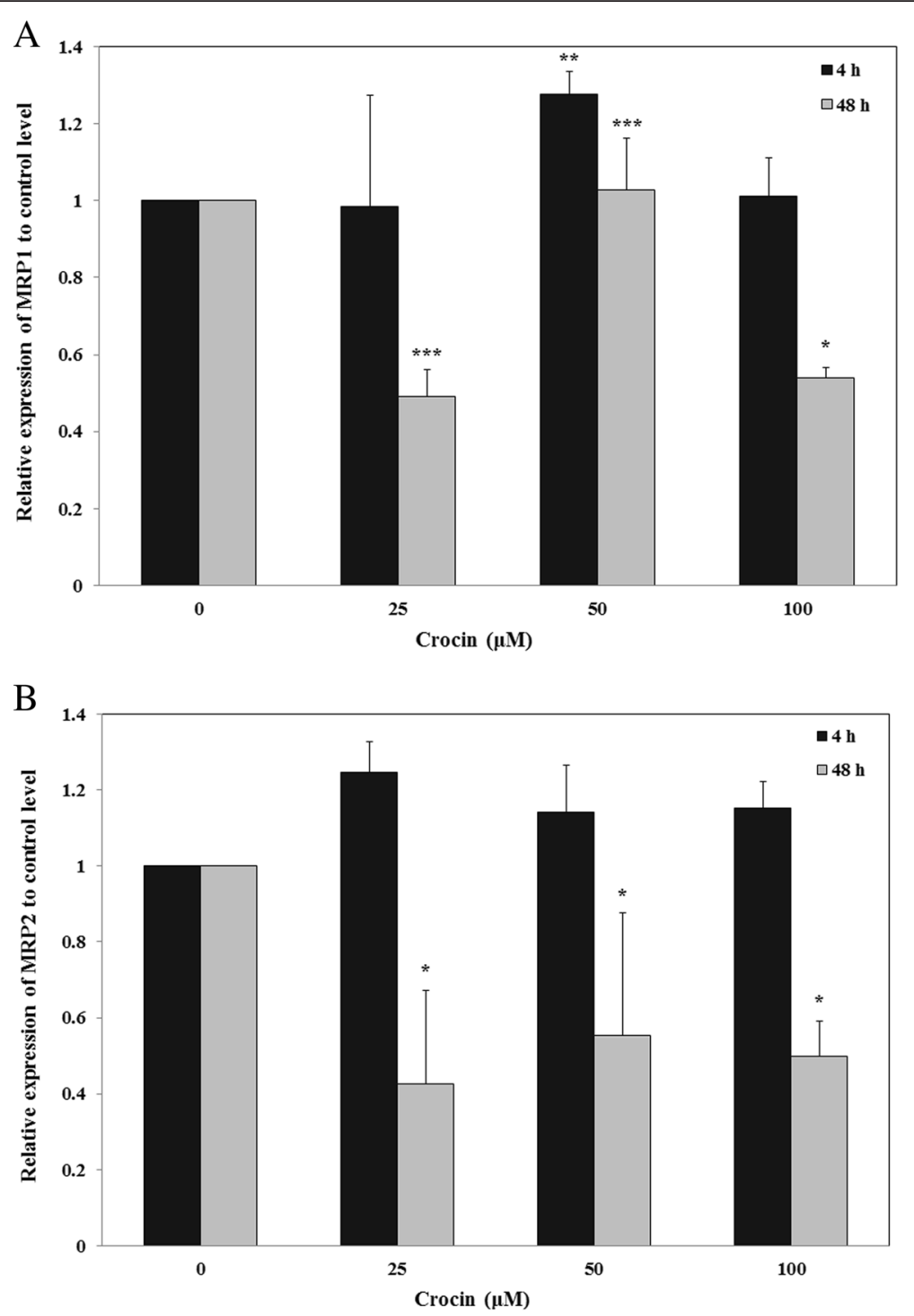

Fig. 3 The effects of crocin on the levels of MRP1 (a) and (b) mRNA in the A2780/RCIS cell line. Cells were treated for 4 and $48 \mathrm{~h}$ with crocin $(0-100 \mu \mathrm{M})$, and MRP1 and MRP2 mRNA expressions were measured by real-time RT-PCR using total RNA extracted from control and treated cells. Values were normalized to the $\beta$-actin content of the samples. The results were expressed as the target/reference ratio of the treated samples divided by the target/reference ratio of the untreated control sample and expressed as mean $\pm \operatorname{SD}(n=3) ;{ }^{*}, p<0.05 ;{ }^{* *}, p<0.01 ;{ }^{* *}, p<0.001$

which are expressed on the surface of cells and pumps chemotherapy drugs out of the cell. In recent years, scientists have tried to find efficient inhibitors of special ABC transporters to overcome MDR phenomenon [27]. Crocin, a major constitute of saffron [10], has shown anticancer activities against several cancer cell lines [19-23], and could be used clinically for the prevention and treatment of cancer in the near future. In this study, we evaluated the effects of crocin on the expression and function of MRP1 and MRP2 in the human ovarian carcinoma cell line A2780 and MRP2-overexpressing cell line A2780/RCIS.
It has been previously shown that induction of the MRP2 expression in human cancer cell lines enhances the resistance to doxorubicin [28], while down regulation of MRP2 enhances cell sensitivity to doxorubicin [29]. Based on these studies and similar studies for MRP1, doxorubicin has been introduced as a substrate of MRP1 and MRP2 transporters [30]. The results of the present study have shown that the MRP1 and MRP2 expression level in the cisplatin-resistant A2780/RCIS cell line was 1.29 and 13 times more than its expression level in its parental sensitive cell line A2780, respectively. On the other hand, doxorubicin had anti-proliferative effects 

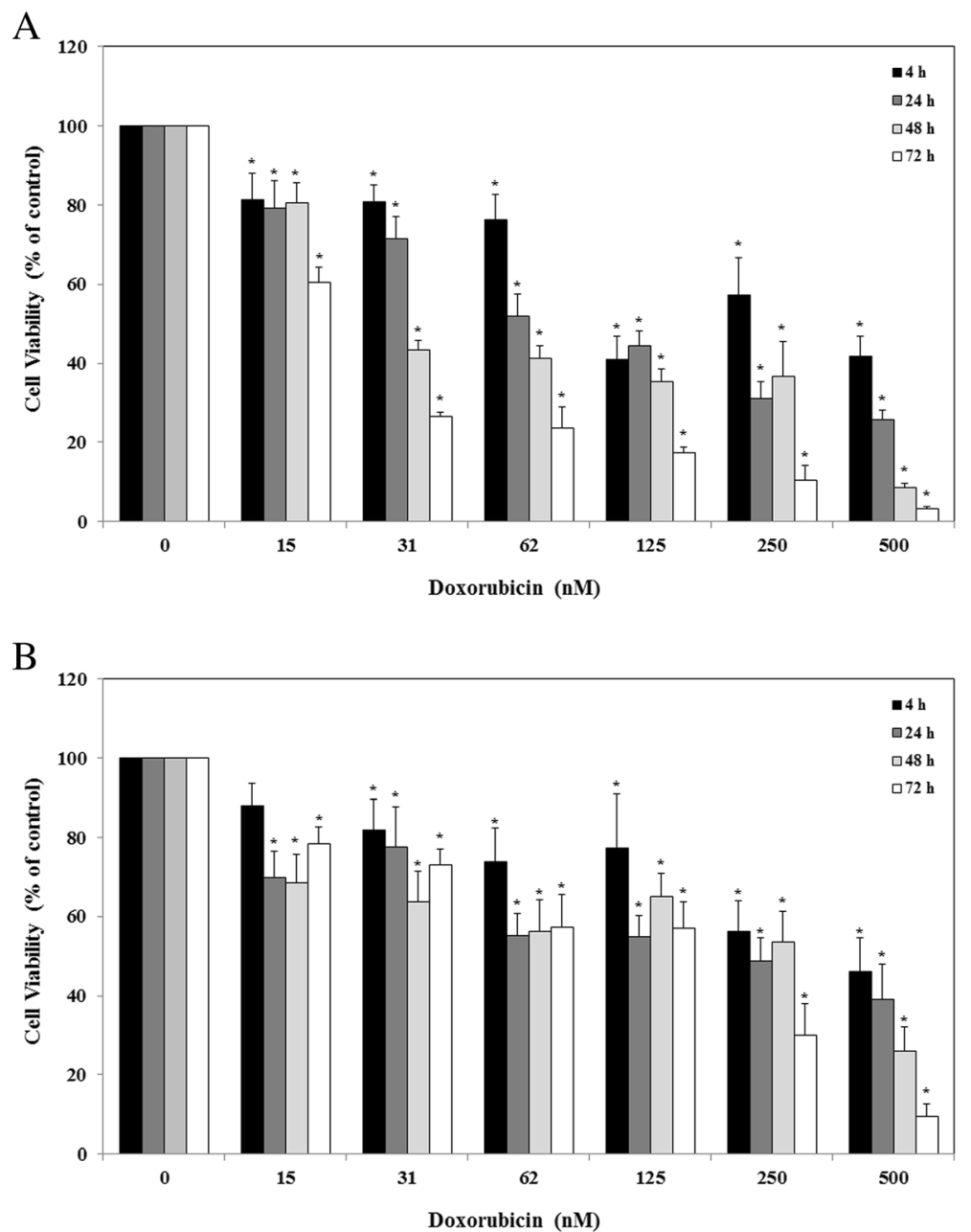

Fig. 4 The effects of doxorubicin on cell viability of A2780 (a) and A2780/RCIS (b) cell lines. The cells were incubated with different concentrations of doxorubicin (0-500 nM) for 4, 24, 48 and $72 \mathrm{~h}$. Cell viability was measured by the MTT assay. Each experiment was repeated independently three times in triplicate tests and data are shown as mean \pm SD. ${ }^{*} P \leq 0.001$

on the A2780 cells with higher intensity when compared with the A2780/RCIS cell line. These results indicated that the higher expression of MRP transporters in A2780/RCIS was accountable for the higher efflux of doxorubicin that resulted to its lower intracellular concentration and lower anti-proliferative activity in this drug-resistant cell line. Interestingly, crocin has shown a similar inhibitory pattern on cell growth of tested cell lines in which its anti-proliferative activity had less potency in A2780/RCIS in compared with the parental sensitive cell line. Totally, these results suggest that crocin could be a substrate of MRP transporters.

Several studies have investigated the molecular mechanism of anticancer activities of crocin against different human cancer cell lines. In one study, the differentially expressed gene of the bladder cancer T24 cell line after treatment with or without crocin has been evaluated using the cDNA microarray. The results showed that under crocin treatment, 836 genes were up-regulated or down-regulated, which were replication factor or were involved in cell cycle controlling and DNA cell apoptosis [31]. Similarly, other studies have shown that crocin could arrest the tumor cell cycle and induce apoptosis by inhibition of the expression of Bcl-2, survivin, cyclin D1, and lactate dehydrogenase A (LDHA) or by upregulation the expression and activity of Bax and nuclear factor erythroid 2-related factor 2 (Nrf2), and by inhibition of the telomerase activity [10, 19, 20, 32, 33]. The investigation of cellular targets of crocin using proteomic screening showed that crocin physically binds to a wide range of cellular proteins such as membrane transporters and enzymes involved in ATP and redox homeostasis [34]. In another study, researchers investigated the inhibition ability of a selection of carotenoids including $\beta$-carotene, crocin, retinoic acid, canthaxanthin, and fucoxanthin on the P-glycoprotein (P-gp; MDR1). These 
Table 1 The effects of different concentrations of crocin on the cell survival percentages of A2780/RCIS cell lines under treatment with doxorubicin (mean \pm SEM)

\begin{tabular}{|c|c|c|c|c|c|}
\hline \multirow[t]{2}{*}{ Time (h) } & \multirow[t]{2}{*}{ Dox $(n M)$} & \multicolumn{4}{|l|}{ Crocin $(\mu \mathrm{M})$} \\
\hline & & 0 & 25 & 50 & 100 \\
\hline \multirow[t]{5}{*}{$4 \mathrm{~h}$} & 16 & $97.04 \pm 2.18$ & $97.39 \pm 10.06$ & $95.88 \pm 3.49$ & $98.77 \pm 3.05$ \\
\hline & 62 & $92.19 \pm 4.94$ & $96.51 \pm 2.36$ & $92.57 \pm 0.70$ & $95.91 \pm 1.34$ \\
\hline & 125 & $91.52 \pm 3.92$ & $95.35 \pm 0.91$ & $91.77 \pm 4.48$ & $92.74 \pm 2.33$ \\
\hline & 250 & $93.56 \pm 2.70$ & $95.13 \pm 4.03$ & $83.37 \pm 5.05$ & $84.1 \pm 2.48$ \\
\hline & 500 & $77.64 \pm 4.70$ & $91.01 \pm 9.06$ & $80.95 \pm 4.23$ & $86.59 \pm 3.93$ \\
\hline \multirow[t]{5}{*}{$24 \mathrm{~h}$} & 16 & $95.54 \pm 2.16$ & $88.37 \pm 1.55$ & $96.30 \pm 1.78$ & $87.02 \pm 6.36$ \\
\hline & 62 & $96.98 \pm 2.80$ & $95.83 \pm 2.48$ & $96.94 \pm 1.63$ & $93.58 \pm 1.41$ \\
\hline & 125 & $96.95 \pm 1.31$ & $92.23 \pm 0.51$ & $96.33 \pm 3.51$ & $75.37 \pm 5.73$ \\
\hline & 250 & $94.75 \pm 2.33$ & $75.84 \pm 4.77$ & $51.68 \pm 4.70^{* * *}$ & $73.80 \pm 5.33$ \\
\hline & 500 & $51.08 \pm 1.17$ & $26.58 \pm 4.38^{* * *}$ & $35.35 \pm 8.88^{* * *}$ & $23.06 \pm 7.58^{* * *}$ \\
\hline \multirow[t]{5}{*}{$48 \mathrm{~h}$} & 16 & $99.39 \pm 2.57$ & $85.17 \pm 3.68$ & $80.87 \pm 5.94^{*}$ & $53.66 \pm 8.40^{* * *}$ \\
\hline & 62 & $95.07 \pm 3.99$ & $73.01 \pm 3.41^{* * *}$ & $60.60 \pm 2.85^{* * *}$ & $54.96 \pm 4.94^{* * *}$ \\
\hline & 125 & $83.10 \pm 2.02$ & $71.49 \pm 2.09$ & $31.12 \pm 8.98^{* * *}$ & $44.45 \pm 6.49^{* * *}$ \\
\hline & 250 & $53.04 \pm 1.34$ & $53.86 \pm 1.73$ & $25.77 \pm 5.78^{* * *}$ & $7.219 \pm 0.14^{* * *}$ \\
\hline & 500 & $32.68 \pm 3.40$ & $19.11 \pm 1.95$ & $8.149 \pm 3.16^{* * *}$ & $3.85 \pm 0.29^{* * *}$ \\
\hline \multirow[t]{5}{*}{$72 \mathrm{~h}$} & 16 & $96.68 \pm 3.55$ & $84.31 \pm 2.84$ & $63.00 \pm 1.68$ & $63.29 \pm 3.29^{* *}$ \\
\hline & 62 & $79.20 \pm 3.88$ & $65.62 \pm 2.16$ & $54.11 \pm 5.12^{* * *}$ & $55.52 \pm 1.85^{* * *}$ \\
\hline & 125 & $60.52 \pm 2.88$ & $53.58 \pm 1.63$ & $47.77 \pm 4.52^{* * *}$ & $34.41 \pm 3.53^{* * *}$ \\
\hline & 250 & $50.13 \pm 2.80$ & $33.17 \pm 2.96^{* *}$ & $34.49 \pm 3.05^{* * *}$ & $28.09 \pm 2.30^{* * * *}$ \\
\hline & 500 & $36.55 \pm 2.82$ & $22.21 \pm 1.16$ & $22.66 \pm 1.71$ & $9.27 \pm 0.93^{* * *}$ \\
\hline
\end{tabular}

Note: The results of the LSD test, which compared the effects of all crocin concentrations on the toxicity of each of the various concentrations of doxorubicin during $4,24,48$, and 72 h. ${ }^{*}, p<0.05 ;{ }^{* *}, p<0.01 ;{ }^{* *}, p<0.001$

carotenoids decreased P-gp mRNA expression levels; increased accumulation of cytotoxic agents which are P-gp substrates that cause to enhance their cytotoxicity effects. Totally, they concluded that carotenoids could be used as adjuvants which are chemosensitizer in chemotherapy [35].

In this study, we aimed to evaluate the effects of crocin on MRP transporter expression and function. For this purpose the sensitivity of tested cell lines to doxorubicin in the presence or absence of non-toxic concentrations of crocin were studied. The MRP activity assay has been designed in two ways, short time exposure with crocin to evaluate the direct interaction between crocin and existing active transporters, and longtime exposure with crocin to evaluate the indirect effect of crocin on the transporter activity as a result of its modifications on the expression level. Interestingly, crocin could not change the A2780/RCIS cell sensitivity to doxorubicin in $4 \mathrm{~h}$, while it significantly increased doxorubicin cytotoxicity after 48 and $72 \mathrm{~h}$ in a concentration-dependent manner in the drug-resistant A2780/RCIS cell line, but not in parental drug-sensitive A2780 cells. On the other hand, the real-time PCR results showed that crocin significantly reduced the
mRNA expression level of MRP1 and MRP2 in A2780/ RCIS cells. Totally, these results indicated that crocin could suppress drug resistance via down regulation of MRP gene expression in the human ovarian cancer resistance cell line.

\section{Conclusion}

In this study we aimed to investigate the ability of crocin on inhibiting multidrug resistance in human ovarian cancer cells by interfering with MRP 1 and MRP2 transporters. The results showed that crocin could decrease the gene expression of MRP1 and MRP2 and exert MDR reversal, and enhance the cytotoxicity of doxorubicin in the human MRP2 overexpressing cell line A2780/RCIS. This study suggests that the application of crocin in combination with chemotherapeutics in cancer treatment could be an effective method to improve the efficacy of chemotherapy and moderate the impact of MDR.

\section{Abbreviations}

ABC, ATP-binding Cassette Transporters; DMSO, Dimethyl Sulfoxide; FBS, Fetal Bovine Serum; LDHA, Lactate Dehydrogenase A; LPS, Lipopolysaccharides; MDR, Multidrug Resistance; MRP, Multidrug Resistance-associated Protein MTT, 3-(4, 5-dimethylthiazol-2-yl)-2, 5-diphenyl tetrazolium bromide; Nrf2, 
Nuclear Erythroid 2-related Factor 2; RCIS, Cisplatin-Resistant Derivative; Real-time RT-PCR, Real-rime Reverse Transcription Polymerase Chain Reaction; ROS, Reactive Oxygen Species; PBS, Phosphate-Buffered Saline; P-gp, P-glycoprotein

\section{Acknowledgments}

The authors are indebted to the Research Council of Mashhad University of Medical Sciences, Iran, for approval and financial support of this project.

\section{Authors' contributions}

FK conceived of the study, designed the study, and coordination and helped to statistical analysis and draft the manuscript. SM carried out the molecular studies, performed the statistical analysis and drafted the manuscript. SA participated in molecular studies. GK, JB and HL conceived of the study and participated in its design. All authors read and approved the final manuscript

\section{Competing interests}

The authors declare that they have no competing interests.

\section{Author details}

'Department of Cell and Molecular Biology, Kish International Campus, University of Tehran, Kish, Iran. ${ }^{2}$ Medical Toxicology Research Center, School of Pharmacy, Mashhad University of Medical Sciences, Mashhad, Iran. ${ }^{3}$ Biotechnology Research Center, School of Pharmacy, Mashhad University of Medical Sciences, P. O. Box 91775-1365, Mashhad, Iran. ${ }^{4}$ Department of Pharmaceutical Biotechnology, School of Pharmacy, Mashhad University of Medical Sciences, Mashhad, Iran. ${ }^{5}$ Institute of Pathology, Charite University, Campus Mitte, Humboldt, Berlin, Germany. ${ }^{6}$ Medical Genetic Research Center, Mashhad University of Medical Sciences, Mashhad, Iran.

\section{Received: 26 November 2015 Accepted: 17 June 2016} Published online: 24 June 2016

\section{References}

1. Ferlay J, Soerjomataram I, Dikshit R, Eser S, Mathers C, Rebelo M, et al. Cancer incidence and mortality worldwide: sources, methods and major patterns in GLOBOCAN 2012. Int J Cancer. 2015;136(5):E359-86.

2. Mitchison D. How drug resistance emerges as a result of poor compliance during short course chemotherapy for tuberculosis [Counterpoint]. Int J Tuberc Lung Dis. 1998;2(1):10-5.

3. Krishna R, Mayer LD. Multidrug resistance (MDR) in cancer: mechanisms, reversal using modulators of MDR and the role of MDR modulators in influencing the pharmacokinetics of anticancer drugs. Eur J Pharm Sci. 2000 11(4):265-83.

4. Choi $\mathrm{C}-\mathrm{H}$. ABC transporters as multidrug resistance mechanisms and the development of chemosensitizers for their reversal. Cancer Cell Int. 2005 5(1):30.

5. Eckford PD, Sharom FJ. ABC efflux pump-based resistance to chemotherapy drugs. Chem Rev. 2009;109(7):2989-3011.

6. Staud F, Pavek P. Breast cancer resistance protein (BCRP/ABCG2). Int J Biochem Cell Biol. 2005;37(4):720-5.

7. Bemis DLCJ, Costello JE, Vorys GC, Katz AE, Buttyan R. The use of herbal and over-the-counter dietary supplements for the prevention of prostate cancer. Curr Urol Rep. 2006;7(3):166-74

8. Hemalswarya SDM. Potential synergism of natural products in the treatment of cancer. Phytother Res. 2006;20(4):239-49.

9. Abdullaev FIE-AJ. Biomedical properties of saffron and its potential use in cancer therapy and chemoprevention trials. Cancer Detect Prev. 2004;6(28):426-32.

10. Kim SH, Lee JM, Kim SC, Park CB, Lee PC. Proposed cytotoxic mechanisms of the saffron carotenoids crocin and crocetin on cancer cell lines. Biochem Cell Biol. 2014;92(2):105-11.

11. Wu Y, Pan R, Geng P. The effect of Crocin against hypoxia damage of myocardial cell and its mechanism. Chinese J Appl Physiol. 2010;26(4):453-7.

12. Asdaq SM, Inamdar MN. Potential of Crocus sativus (saffron) and its constituent, crocin, as hypolipidemic and antioxidant in rats. Appl Biochem Biotechnol. 2010;162(2):358-72.

13. Ordoudi SA, Befani CD, Nenadis N, Koliakos GG, Tsimidou MZ. Further examination of antiradical properties of Crocus sativus stigmas extract rich in crocins. J Agric Food Chem. 2009;57(8):3080-6.
14. Xu GL, Yu SQ, Gong ZN, Zhang SQ. Study of the effect of crocin on rat experimental hyperlipemia and the underlying mechanisms. Zhongguo Zhong Yao Za Zhi. 2005;30(5):369-72.

15. He SY, Qian ZY, Tang FT, Wen N, Xu GL, Sheng L. Effect of crocin on experimental atherosclerosis in quails and its mechanisms. Life Sci. 2005; 77(8):907-21.

16. Wang Y, Han T, Zhu Y, Zheng CJ, Ming QL, Rahman K, et al. Antidepressant properties of bioactive fractions from the extract of Crocus sativus $L$. J Nat Med. 2010;64(1):24-30.

17. Nam KN, Park YM, Jung HJ, Lee JY, Min BD, Park SU, et al. Anti-inflammatory effects of crocin and crocetin in rat brain microglial cells. Eur J Pharmacol. 2010;648(1-3):110-6

18. Xu GL, Li G, Ma HP, Zhong H, Liu F, Ao GZ. Preventive effect of crocin in inflamed animals and in LPS-challenged RAW 264.7 cells. J Agric Food Chem. 2009:57(18):8325-30.

19. Zhao P, Luo CL, Wu XH, Hu HB, Lv CF, Ji HY. Proliferation apoptotic influence of crocin on human bladder cancer T24 cell line. Zhongguo Zhong Yao Za Zhi. 2008;33(15):1869-73.

20. Xu HJ, Zhong R, Zhao YX, Li XR, Lu Y, Song AQ, et al. Proliferative inhibition and apoptotic induction effects of crocin on human leukemia HL-60 cells and their mechanisms. Zhongguo Shi Yan Xue Ye Xue Za Zhi. 2010;18(4):887-92.

21. Chryssanthi DG, Lamari FN, latrou G, Pylara A, Karamanos NK, Cordopatis P. Inhibition of breast cancer cell proliferation by style constituents of different Crocus species. Anticancer Res. 2007:27(1A):357-62.

22. Aung HH, Wang CZ, Ni M, Fishbein A, Mehendale SR, Xie JT, et al. Crocin from Crocus sativus possesses significant anti-proliferation effects on human colorectal cancer cells. Exp Oncol. 2007;29(3):175-80.

23. Garcia-Olmo DC, Riese HH, Escribano J, Ontanon J, Fernandez JA, Atienzar $M$, et al. Effects of long-term treatment of colon adenocarcinoma with crocin, a carotenoid from saffron (Crocus sativus L.): an experimental study in the rat. Nutr Cancer. 1999:35(2):120-6.

24. Mosaffa F, Lage H, Afshari JT, Behravan J. Interleukin-1 beta and tumor necrosis factor-alpha increase ABCG2 expression in MCF-7 breast carcinoma cell line and its mitoxantrone-resistant derivative, MCF-7/MX. Inflamm Res. 2009:58(10):669-76.

25. Tatebe S, Sinicrope FA, Kuo MT. Induction of multidrug resistance proteins MRP1 and MRP3 and gamma-glutamylcysteine synthetase gene expression by nonsteroidal anti-inflammatory drugs in human colon cancer cells. Biochem Biophys Res Commun. 2002;290(5):1427-33.

26. Hadizadeh F, Mohajeri SA, Seifi M. Extraction and purification of crocin from saffron stigmas employing a simple and efficient crystallization method. PJBS. 2010;13(14):691-8.

27. Ozben T. Mechanisms and strategies to overcome multiple drug resistance in cancer. FEBS Lett. 2006;580(12):2903-9.

28. Cui Y, Konig J, Buchholz JK, Spring H, Leier I, Keppler D. Drug resistance and ATP-dependent conjugate transport mediated by the apical multidrug resistance protein, $M R P 2$, permanently expressed in human and canine cells. Mol Pharm. 1999:55(5):929-37.

29. Koike K, Kawabe T, Tanaka T, Toh S, Uchiumi T, Wada M, et al. A canalicular multispecific organic anion transporter (CMOAT) antisense CDNA enhances drug sensitivity in human hepatic cancer cells. Cancer Res. 1997;57(24):5475-9.

30. Borst P, Evers R, Kool M, Wijnholds J. A family of drug transporters: the multidrug resistance-associated proteins. J Natl Cancer Inst. 2000;92(16):1295-302.

31. LV CF, Luo $C L$, Ji HY, Zhao P. Influence of crocin on gene expression profile of human bladder cancer cell lines T24. Zhongguo Zhong Yao Za Zhi. 2008; 33(13):1612-7.

32. D'Alessandro AM, Mancini A, Lizzi AR, De Simone A, Marroccella CE, Gravina $G L$, et al. Crocus sativus stigma extract and its major constituent crocin possess significant antiproliferative properties against human prostate cancer. Nutr Cancer. 2013;65(6):930-42.

33. Noureini SK, Wink M. Antiproliferative effects of crocin in HepG2 cells by telomerase inhibition and hTERT down-regulation. APJCP. 2012;13(5):2305-9.

34. Hosseinzadeh H, Mehri S, Heshmati A, Ramezani M, Sahebkar A, Abnous K. Proteomic screening of molecular targets of crocin. Daru. 2014;22(1):5.

35. Eid SY, El-Readi MZ, Wink M. Carotenoids reverse multidrug resistance in cancer cells by interfering with ABC-transporters. Phytomedicine. 2012; 19(11):977-87. 